\title{
Effects of stress on risk taking'
}

AMIA LIEBLICH

THE HEBREW UNIVERSITY OF JERUSALEM

Risk-taking behavior was measured under three experimental conditions: (A) a neutral condition; (B) irrelevant stress condition; and $(C)$ relevant stress condition. It was found that the amount of risk taking tended to increase under both stress conditions.

The term "risk taking" usually refers to a decision that has uncertain outcomes with a prospect of loss or failure. Kogan \& Wallach (1967), mentioned the lack of research about the influence of external situations on risk-taking behavior. This study attempts to compare the amount of risk taken by Ss under relaxed and stress conditions.

Two kinds of stress were manipulated: relevant and irrelevant stress. Relevant stress was defined as a stress condition which the person perceives as depending on his performance on the required task; he believes that he can reduce the amount of stress as a result of his behavior. In the irrelevant stress condition the person believes that the amount of stress is a priori fixed, and that his performance on the task will not influence the amount of stress which he suffers. This study investigated the effect of these two kinds of stress on the amount of risk taken by the individual.

\section{Subjects}

Two groups of female first year students at the university participated in the experiment. Each group consisted of 25 Ss. Group 1 was tested twice, in two different experimental conditions, the second administration being conducted six months after the first. Group 2 was generally matched to Group 1, and was tested under only one experimental condition. Instruments

An individual test, introduced by Minkowich (1964), was conducted to measure risk-taking tendency. The test consisted of three series of bets on cards randomly drawn from a pack of 48 playing cards (aces and jokers were removed). In the first series (Part 1), $S$ guessed 10 times with no feedback within the series, and with no monetary reward. The $S$ guessed by choosing in each trial one of the six following probabilities of success: $0.75,0.50,0.25,0.17,0.125$ and $0.085-$ and predicting the type or name of the card that would be drawn. At the end of the series 10 cards were drawn and the $S$ marked the number of her hits.

The second series of 10 trials (Part 2) differed from the first in two respects: feedback was given after each trial, and monetary bets were included in each alternative. A sum of two Israel pounds was given to each $\mathrm{S}$, and she was told that every right guess would earn her a known additional sumwhereas every wrong guess would cost her a known sum from the amount she had. The total sum remaining in the S's possession at the end of the game would be hers. The size of the bets was fixed such that the possible gain was bigger when the probability of success was lower. The expected value of this gain was positive when the probability of success was 0.75 , zero for the probability of 0.50 , and negative and descending for the other probabilities.

The third series (Part 3) was comprised of five decisions, all of which involved only the choice of the bet. The probability of success was fixed $(0.25)$, and the Ss could choose the sum they were willing to risk from eight possible sums. This chosen sum would be gained or lost as an outcome of S's success or failure in her guess.

An index of risk was computed separately for the three parts of the test. In Parts 1 and 2 scores from 1 to 6 were assigned to the chosen probability from the highest $(0.75)$ to the lowest $(0.085)$ probability respectively. In Part 3 a score of 1 was assigned to the lowest bet, and 8 to the highest bet.

An electrical shocker described by Lieblich (1966) was used for creating stress.

Procedure

Neutral condition. The guessing game was carefully explained to the $S$, and the test was conducted individually. Twenty-five $\mathrm{Ss}$ participated in this condition.

Irrelevant stress condition. The Ss were introduced to a "physiology laboratory." The guessing game was carefully explained, a short interview was conducted about S's health, and the electrodes connected to S's wrist. The instructions given after this (translated verbatim from Hebrew) were:

"During the test you will get electrical shocks a few times. The shocks will be of various intensities. Pay attention, I will give you one shock now. (One shock of standard intensity was given to S.) This is one of the intensities that will appear in the test. There is no relation whatsoever between your behavior in the guessing game and the number or intensity of the shocks you will get. The schedule of the shocks was planned beforehand. During the test I will not inform you when you will get a shock."

After the instructions the guessing game was conducted as in Condition (A). All Ss were shocked in a fixed schedule on certain trials of the test.

Relevant stress condition. This condition differed from the former in only one point of the instructions 
Table 1. Means and variances of risk-taking scores for neutral, irrelevant stress and relevant stress conditions.

\begin{tabular}{llllll} 
& \multicolumn{5}{c}{ Risk-taking scores } \\
Condition & & Part 1 & Part 2 & Part 3 & N \\
\hline A. Neutral & $\overline{\mathrm{X}}$ & 26.68 & 27.80 & 20.06 & 25 \\
& $\mathrm{~S}^{2}$ & 63.99 & 86.95 & 91.07 & \\
& $\overline{\mathrm{X}}$ & 29.88 & 31.36 & 18.84 & 25 \\
B. Irrelevant & $\mathrm{X}$ & 11.68 & 57.74 & 99.43 & \\
Stress & $\mathrm{S} 2$ & & & \\
C. Relevant & $\overline{\mathrm{X}}$ & 31.56 & 30.00 & 19.44 & 25 \\
Stress & $\mathrm{S}^{2}$ & 32.79 & 48.39 & 66.31 & \\
\hline
\end{tabular}

read to the $S$. After the demonstration of the shock as described in condition B, Ss were told:

"The number of shocks you will get and their intensity depend on your behavior. A certain rule exists which relates your choice in the test to whether the shock will appear or not, and to its intensity. This rule is not simple, but if you succeed and discover it, you will be, naturally, able to reduce the number of shocks given during the game."

A few examples for "rules" were given. One of them was: "You will get a shock every second time you guess a diamond."

The rules used as examples were, intentionally, very complicated. Actually the shocks were applied in the same manner as in Condition (B), i.e., independent of the S's behavior. In this condition 25 new Ss were tested.

\section{Results}

Mean risk-taking scores and their variances were computed separately for the three parts of the test described above, and for the three experimental conditions. The results appear in Table 1.

The results indicated that in Part 1 and 2 of the test the tendency to risk was higher under stress conditions than in the neutral condition. In part 3 the tendency to risk seemed lower under stress, but the difference was smaller. For all parts, no consistent differences appeared between the two stress conditions.

Significance of the differences was computed by Wilcoxon Test for the comparison of Conditions (A) and (B), which are statistically dependent, and by the Mann-Whitney $U$ Test for the comparison of Conditions (A) and $(C)$, which are statistically independent (Siegel, 1956). For Parts 1 and 2 of the test all the computed differences were found to be significant $(\alpha<.05)$ except for the difference between neutral and relevant stress condition in Part 2. For Part 3 of the risk-taking test none of the differences were statistically significant.

As to the variances of the risk-taking scores, which appear in Table 1, a consistent tendency, mostly significant by the F-Test, showed that the variance of risk-taking was lower under both stress conditions than in the neutral condition.

\section{Discussion}

The results of the study tended, in general, to indicate that under stress mean risk-taking was higher and the variance lower than in the neutral condition. No difference was found between relevant and irrelevant stress.

It was surprising not to find any consistent differences between the conditions of relevant and irrelevant stress. It could be assumed that relevant stress would be more motivating. In fact, an interesting difference was found between relevant and irrelevant stress conditions, as explained below.

A score of vacillations in the choice of each individual was computed, based on the number of changes in the decisions of an individual within a series in the test. No significant differences in vacillations were found between the neutral condition and the irrelevant stress condition. On the other hand, the mean number of vacillations was significantly higher $(p<.01)$ in the decisions made under relevant than under the irrelevant stress condition. These findings indicate that in the relevant stress condition Ss tried to find the rule by which they would avoid the electrical shocks, and these attempts highly enhanced the number of their vacillations.

As indicated above, the results of Part 3 in the risk-taking test deviated from the main pattern. An explanation of this fact might be the reduction of the intensity of stress towards the end of the session. It is possible that some habituation took place, and that anxiety decreased with time. Moreover, Part 3 was printed on the last page of the booklet, so that Ss perceived it was an end-game, a fact which might have affected their behavior. Another experiment, in which the order of the parts of the test will be rotated, is now planned to investigate this point.

\section{References}

KOGAN, N., \& WALLACH, A. W. Risk taking as a function of the situation, the person and the group. In G. Mandler and P. Mussen (Eds.), New directions in psychology III. New York: Holt, Rinehart \& Winston, 1967.

LIEBLICH, AMIA. Effects of psychological stress on risk taking. Unpublished doctoral dissertation, The Hebrew University of Jerusalem, 1966.

MINKOWICH, A. Correlates of ambivalence, risk-taking and rigidity. Scientific report 1, AF AOER Grant 63-62, Jerusalem, 1964.

SIEGEL, S. Nonparametric statistics for the behavioral sciences. New York: McGraw-Hill, 1956

Note

1. This study is based on a Ph.D. dissertation conducted under the supervision of Dr. A. Minkowich, and approved by the Hebrew University of Jerusalem in June 1967. The author is grateful to Dr. Abram Minkowich for his advice. The study was supported by the United States Air Force, Grant AF EOAR 65-32. 